\title{
A new counter-intuitive therapy for adult amblyopia
}

2

3 Lunghi Claudia, $\mathrm{PhD}^{1,2}$, Sframeli Angela Tindara $\mathrm{MD}^{3}$, Lepri Antonio $\mathrm{MD}^{3}$, Lepri Martina $\mathrm{MS}^{3}$, Lisi

Affiliations: ${ }^{1}$ Department of Translational Research on New Technologies in Medicine and

7 Surgery, University of Pisa, Italy; ${ }^{2}$ Laboratoire des systèmes perceptifs, Département d'études

8

9 cognitives, École normale supérieure, PSL University, CNRS, 75005 Paris, France; ${ }^{3}$ Ophthalmology

Unit, Deptartment of Surgical, Medical, Molecular and Critical Area Pathology, University of Pisa,

Italy; ${ }^{4}$ Neuroscience Institute, National Research Council (CNR), Pisa, Italy; ${ }^{5}$ IRCCS Stella Maris, Calambrone, Pisa, Italy.

${ }^{+}$Co-senior authors

\section{*Corresponding Author:}

Alessandro Sale

Neuroscience Institute, National Research Council (CNR), Via Moruzzi 1, 56124, Pisa, Italy;

Email: sale@in.cnr.it

Manuscript information

\# Characters: title $=51$, running head $=33$.

\# Words: abstract $=156$, introduction $=457$, discussion $=705$, body $=3247$

\# Figures: 5

\# Color Figures: 0 


\section{Abstract}

Visual cortex plasticity is high during a critical period of early postnatal development, but rapidly diminishes with the transition to adulthood. Accordingly, visual disorders such as amblyopia (lazy eye), can be treated early in life by long-term occlusion of the non-amblyopic eye, but may become irreversible in adults, because of the decline in brain plasticity. Here we show that a novel counter-intuitive approach can promote the recovery of visual function in adult amblyopic patients: short-term occlusion of the amblyopic (not the fellow) eye, combined with physical exercise (cycling). After six brief ( $2 \mathrm{~h}$ ) training sessions, visual acuity improved in all ten patients $(0.15 \pm 0.02$ LogMar), and six of them also recovered stereopsis. The improvement was preserved for up to one year after training. A control experiment revealed that physical activity was crucial for the recovery of visual acuity and stereopsis. Thus, we propose a non-invasive therapeutic strategy for adult human amblyopia based an inverse-occlusion and physical exercise procedure. 


\section{Introduction}

Amblyopia is a neurodevelopmental disorder of vision ${ }^{1,2}$ with a prevalence ranging from 1 to $5 \%$ depending on the population tested ${ }^{3}$. The most frequent causes of amblyopia are strabismus, anisometropia and deprivation (for example due to cataracts) early in life ${ }^{1,2}$, within the so-called critical period, defined as the temporal window during development where the plastic potential of the visual cortex is maximal ${ }^{4,5}$. If the retinal image is degraded during development, the visual cortex disregards the eye providing degraded input and responds primarily to the eye providing the better signal. Amblyopia can be ameliorated in young children by the long-term occlusion of the better (non-amblyopic) eye ${ }^{3}$. However, in humans, treatment after the closure of the critical period dramatically decreases therapy efficacy requiring extensive occlusion of the non-amblyopic eye to produce only a modest improve of visual acuity (234 hours of occlusion per

0.1 LogMar acuity improvement ${ }^{6}$ ). New therapeutic strategies recently proposed for adult amblyopia involve either very prolonged visual training ${ }^{7-12}$, or invasive manipulations ${ }^{13,14}$, rendering them almost ineffective for clinical purposes.

Recently, new hope has emerged from evidence that the early visual cortex retains some degree of plasticity in adult humans ${ }^{15-23}$. Many studies have demonstrated a plastic response to visual deprivation: a few hours ${ }^{15,16}$ or a few days ${ }^{17,18}$ of binocular deprivation modulate excitability of the primary visual cortex. But also monocular occlusion of one eye for a few hours paradoxically boosts the deprived eye signal, shifting ocular dominance in favor of the deprived eye ${ }^{19-22}$. This counter-intuitive effect of monocular deprivation reflects a compensatory reaction of the visual cortex to deprivation aimed at maintaining the average cortical activity constant, indicating that some form of homeostatic plasticity ${ }^{24}$ is also present in adult humans. Importantly, this plasticity is mediated by a decrease of GABA concentration in the primary visual cortex ${ }^{25}$ and is enhanced by physical exercise ${ }^{26}$. 
recent studies on animal models ${ }^{27-30}$ : in adult rats and mice physical activity boosts visual cortical plasticity by modulating the levels of GABAergic inhibition ${ }^{27-29}$ via a specific somatosensory- visual circuitry $^{27-30}$. Interestingly, visual cortical GABAergic interneurons are implicated in the mechanisms controlling the onset and offset of the visual critical period in these animal models ${ }^{31}$. strategy for adult amblyopia, no attempts have been made, to date, to investigate its effects on amblyopic human subjects. Focusing on adult anisometropic patients, we performed a counterintuitive experiment which combined short-term deprivation of the amblyopic eye with physical exercise. We found that after six short training sessions ( $2 \mathrm{~h}$ each), visual acuity and stereopsis improved in adult patients, and that the improvement persisted for up to one year after the end of the treatment.

\section{Methods}

\section{Subjects}

Adult anisometropic patients were enrolled for the study after an ophthalmic screening examination in which detailed ocular and systemic anamnesis has been investigated (see been excluded from the study as long as patients presenting any chronic systemic disease. excluded. 


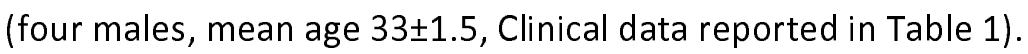

89

\section{Ethical Statement}

Experimental procedures are in line with the declaration of Helsinki and were approved by the regional ethics committee [Comitato Etico Pediatrico Regionale-Azienda OspedalieroUniversitaria Meyer-Firenze (FI)], under the protocol “Plasticità del sistema visivo" (3/2011).

\section{Apparatus and Stimuli}

\section{Binocular Rivalry}

The experiment took place in a dark and quiet room. Visual stimuli were generated by the ViSaGe stimulus generator (CRS, Cambridge Research Systems), housed in a PC (Dell) controlled by Matlab programs. Visual stimuli were two Gaussian-vignetted sinusoidal gratings (Gabor Patches), oriented either $45^{\circ}$ clockwise or counterclockwise (size: $2 \sigma=2^{\circ}$, spatial frequency: $2 \mathrm{cpd}$ ), presented on a uniform background (luminance: $37.4 \mathrm{~cd} / \mathrm{m} 2$, C.I.E: 0.4420 .537 ) in central vision with a central black fixation point and a common squared frame to facilitate dichoptic fusion. Visual stimuli were displayed on a 20-inch Clinton Monoray (Richardson Electronics Ltd., LaFox, IL) monochrome monitor, driven at a resolution of $1024 \times 600$ pixels, with a refresh rate of $120 \mathrm{~Hz}$. Observers viewed the display at a distance of $57 \mathrm{~cm}$ through CRS Ferro-Magnetic shutter goggles that occluded alternately one of the two eyes each frame. Responses were recorded through the computer keyboard.

\section{Procedures}

Screening Examination 

administrations) in order to register cycloplegic ametropia with an autorefractometer (Topcon last eye drop administration. During the screening visit, all patients underwent non-contact ocular biometry (IOLMaster 500, Carl Zeiss, Germany) and axial length (AL), anterior chamber depth (ACD) and white to white signal (WTW) have been registered. A complete examination of ocular motility has also been performed in order to exclude muscular causes of amblyopia: cover and uncover far and near tests, versions and objective convergence evaluation, Irvine test and 8 diopters Paliaga test for microstrabimus. Fixational quality was also assessed using microperimetry

(Nidek MP-1 Professional). The screening examination was completed with endothelial cell count (Tomey EM 3000, Tomey, Germany), corneal topography (Sirius system, CSO, Firenze, Italy) to study keratometric parameters, slit lamp biomicroscopy (SL 9900, CSO, Italy) and complete phundus examination with both 90D and 20D lenses have also been performed. abnormal or unclear ocular findings (example.g., elevated IOP, presence of epiretinal membranes, abnormal ocular motility that may have caused amblyopia. 
Main experiment: each training session lasted $2 \mathrm{~h}$ and consisted in the combination of amblyopic eye occlusion and physical exercise. Occlusion of the amblyopic eye was performed using eyepatching. The eye-patch was custom-made of a translucent plastic material that allowed light to reach the retina (attenuation 15\%) but completely prevented pattern vision, as assessed by the Fourier transform of a natural world image seen through the eye-patch. During the $2 \mathrm{~h}$ of monocular occlusion, patients sat on a stationary bike equipped with a chair and a computer monitoring physical activity parameters (cycling speed, distance) and heart rate through a wireless chest band. Patients were instructed to cycle intermittently maintaining a heart rate between 110 and $120 \mathrm{bpm}$ for 10 minutes, interleaved with 10 minutes rest; the experimenter controlled that physical exercise was performed according to these parameters. A 20" monitor (LG) was placed in front of the bike at a distance of $90 \mathrm{~cm}$, patients watched a movie projected onto the monitor during the training. Before and after each training session, visual acuity (ETDRS charts), stereoacuity (TNO test) and sensory eye dominance (binocular rivalry) were assessed for each patient. This training was repeated six times over a four-week period: on three consecutive days during the first week of training and one day per week during the following three weeks (Figure 1). amblyopic eye (same procedure as main experiment) without simultaneous physical exercise. During the $2 \mathrm{~h}$ of monocular occlusion patients sat at a distance of $90 \mathrm{~cm}$ in front of a $20^{\prime \prime}$ monitor (LG) and watched a movie. This training was repeated three times in three consecutive days. 156 Before and after each training session, visual acuity (ETDRS charts), stereoacuity (TNO test) and sensory eye dominance (binocular rivalry) were assessed for each patient. 
stimulus was associated with which eye until the end of the session, when it was verified visually.

Because of anisometropia, when rivalrous stimuli having equal contrast were presented,

non-amblyopic eye. In order to adjust the monocular stimuli contrast to compensate for the

experimental blocks were performed. At each block, contrast of monocular stimuli was varied,

increasing contrast of stimuli presented to the amblyopic eye (maximum contrast: $100 \%$ ) and 
(complete dominance of the non-amblyopic eye) to 1 (complete dominance of the amblyopic eye),

by computing the contrast between the mean phase duration of the two eyes:

$$
\text { Ocular Dominance Index }=\frac{\text { AmblyopicEye }- \text { NonAmblyopicEye }}{\text { AmblyopicEye }+ \text { NonAmblyopicEye }}
$$

Statistical analyses were performed using SPSS20 and Matlab softwares. BCVA,

Stereoacuity and Sensory Eye Dominance before, during and after training were compared using

\section{Results}

Ten adult anisometropic amblyopes (Table 1), selected for good fixation and with no other neurological deficit, underwent six brief training sessions over a four-week period (three on consecutive days during the first week and one session per week in the following three weeks:

Figure 1). To assure a high homogeneity of the sample, only purely anisometropic amblyopes were 
watching a movie. During the training period, visual acuity (ETDRS charts) significantly improved in all patients (Figure 1B), with a first improvement evident after the first two hours of patching $(t(9)=2.37, p=0.04)$, increasing after each session. The average improvement after the sixth (and last) training session was $0.15 \pm 0.02 \operatorname{LogMar}(t(9)=7.7, p<0.001$, Figure $2 \mathrm{~A}$, Repeated Measures ANOVA, $\left.F(6,54)=24.9, p<0.001, \eta^{2}=0.73\right)$. This improvement was maintained in the follow-up measurements (Figure 3) obtained one month $(t(9)=4.04, p=0.003)$, three months $(t(9)=5.22$, $p<0.001)$ and one year after the end of training $(t(5)=3.81, p=0.012)$. The initial visual acuity of the amblyopic eye and the acuity improvement observed at the end of the training were not correlated (Spearman's rho=-0.16, $p=0.67$, Figure $4 A$ ), indicating that the effect of the training was independent of anisometropia severity.

The training was also effective enough to induce a significant improvement of stereothresholds (TNO test) in six out of the ten patients, independently of their occlusion therapy history (Figure $1 \mathrm{C}$ )., This was a significant improvement for the sample (Repeated Measures ANOVA, $\left.F(6,54)=6.2, p=0.02, \eta^{2}=0.7\right)$. Two of these patients were completely stereoblind before training (Figure $1 \mathrm{C}$ ). The patients who improved stereopsis during training also maintained the improvement in the follow-up measurements up to one year (Figure 3B). Fixation quality of the amblyopic eye did not change after training (Figure 4B, fixations within $2^{\circ}$ pre-training $=$ $84.6 \pm 4.8 \%$, after training $=85.8 \pm 4.9 \%, t(8)=0.26, p=0.8$ ), indicating that the improvement visual function was not attributable to a change in ocular motility. Interestingly, the sensory dominance of the amblyopic eye, measured with a binocular rivalry task, also significantly increased after short-term occlusion (Figure $2 \mathrm{~B}, \mathrm{t}(5)=5.26, \mathrm{p}=0.003$ ), suggesting a change in the binocular neuronal circuitry. The post-training changes in visual acuity, stereo-acuity and ocular dominance did not correlate with each other (all ps>0.77). 
In debriefing questionnaires, all patients reported a qualitative improvement of vision in

the amblyopic eye ("I have a sharper/more contrasted/brighter vision from the amblyopic eye").

Two patients also reported a significant reduction in the occurrence of headaches/migraines

usually experienced after prolonged exposure to screens, three patients reported an improvement to confuse adjacent letters with each other").

$F(3,12)=11.18, p=0.005, \eta^{2}=0.74$, Figure $7 A$ ). However, the visual acuity improvement achieved physical exercise was about double (Bootstrap sign-test, $10^{6}$ repetitions, $p<0.001$, Figure $5 \mathrm{C}$ ) than

Finally, in the control condition stereo-thresholds remained unchanged in all patients (Figure 5B), recovery of visual function in adult amblyopes.

\section{Discussion}

Together, our results show that six brief sessions of short-term deprivation of the 
and stereopsis in adult anisometropic patients. The first surprising aspect of our results is that patching the amblyopic eye is effective in improving visual acuity in anisometropic patients, a procedure that is opposite to the traditional occlusion therapy used for amblyopia ${ }^{3}$. Patching the amblyopic eye is commonly referred to as "inverse occlusion" ${ }^{\prime 32}$ and has been historically used as an alternative treatment for cases of amblyopia with eccentric fixation, aimed at preventing the reinforcement of the eccentric fixation point in the amblyopic eye ${ }^{33-36}$. Even though prolonged inverse occlusion (12 days) was found to be effective to some extent in this particular class of patients, its effect was weaker than the effect of traditional occlusion ${ }^{36}$, and this practice was abandoned. Our results show that short-term inverse occlusion (combined with physical exercise) might be more effective (up to 20 times faster) than traditional occlusion for the recovery of visual function in adult amblyopes. Moreover, the effects of short-term inverse occlusion is not due to a change in ocular motility, as fixation quality of the amblyopic eye did not change after training. Rather, we propose that the effect is due to a genuine boost of the amblyopic eye signal, consistent with the homeostatic plasticity previously observed in adult emmetropes ${ }^{19-22,37,38}$ and amblyopes $^{39,40}$.

Our data show that moderate physical exercise boosts the effect of short-term monocular deprivation, inducing a larger improvement of visual acuity and stereo-sensitivity compared to inverse occlusion alone, and might be crucial to promote the long-term effect of the training. Our findings are consistent with recent evidence that voluntary physical exercise enhances visual cortical activity ${ }^{41}$ and promotes visual plasticity in adult rodents 27,29 and in normally sighted humans $^{26,42,43}$. Our results also show that physical exercise boosts visual cortical plasticity in amblyopic human subjects, after the closure of the critical period. The modulation of visual cortical activity and plasticity by physical activity has been linked to a decrease of GABAergic inhibition in the primary visual cortex of mice and rats $^{28,30}$. We have recently found that a 
276 decrease of GABAergic inhibition in the primary visual cortex is also one of the key mechanisms

277 mediating the effect of short-term monocular deprivation in adult humans: GABA concentration

278 decreases in V1 after $2 \mathrm{~h} 30$ of monocular deprivation and the change in GABA strongly correlates

279 with the change in ocular dominance measured with binocular rivalry ${ }^{37}$. It is therefore plausible

that the beneficial effect of exercise on visual plasticity observed here is mediated by a modulation of GABAergic inhibition, promoting ocular dominance plasticity.

More generally, physical exercise also increases neurotrophic factors (BDNF, IGF-1 and

VEGF) and cardiovascular fitness, two factors that might be involved in mediating

neuroplasticity ${ }^{44}$. Increased BDNF following exercise has been related to enhanced hippocampal

plasticity and neurogenesis ${ }^{45,46}$, as well as with improved memory and executive functions in

humans $^{47}$. Importantly, BDNF is also one of the critical mechanisms underlying visual plasticity,

regulating the critical period for ocular dominance plasticity ${ }^{48-50}$. On the other hand, whereas

cardiovascular fitness has been related to higher cognitive performances, no consistent correlation

between cardiovascular fitness and improved cognitive functions after physical exercise has been

found ${ }^{51}$ and there is no evidence at present of a role of cardiovascular fitness in mediating visual cortical plasticity.

In conclusion, we propose a new training paradigm that is totally non-invasive, does not require extensive supervision, and leaves the patients free to perform pleasant activities such as

is the principal cause of (predominantly monocular) visual loss in the pediatric population

296 (prevalence up to $5 \%^{3}$ ). While we believe that our data can provide a valid therapeutic strategy for adult amblyopic patients and adolescents resilient to the standard occlusion therapy, we are well aware that the methods may be not appropriate as a substitution of standard clinical therapy, 
300

301

302

303

304

305

306

307

308

309

310

311

312

313

314

315

316

317

318

319

320

321

amblyopic children ${ }^{39}$ suggesting that the same method in principle could be used, but given the high plasticity of young children visual cortex it should be first carefully validated with a dosedependent chart.

\section{Acknowledgements}

The authors are grateful to Dr. Roberto Caputo for help with the selection of patients and continual advice, Drs. Aris Dendramis and Anna Bettinelli for help during the data collection and Prof. David Burr for helpful comments throughout the project.

\section{Funding}

The research leading to these results was funded by: the European projects ERA-NET Neuro-DREAM and ECSPLAIN (European Research Council under the Seventh Framework Programme, FPT/2007-2013, grant agreement n. 338866) and the Italian Ministry of University and Research under the project "PRIN 2015".

\section{Authors Contributions}

C.L., A.S. and M.C.M. designed the experiment; A.T.S., A.L., M.L and D.L performed patients' clinical examinations; C.L. collected and analyzed the data; all authors discussed the results; C.L., M.C.M., A.T.S. and A.S. wrote the paper.

\section{Conflict of interest}

The authors declare that there is no conflict of interest regarding the publication of this paper. 
323

\section{References}

1. Holmes JM, Clarke MP. Amblyopia. Lancet 2006;367(9519):1343-1351.

2. Levi DM, Carkeet A. Amblyopia: a consequence of abnormal visual development. In: Simons K, editor. Early Visual Development, Normal and Abnormal. New York, NY: Oxford University Press; 1993 p. 391-408.

3. Webber AL, Wood J. Amblyopia: prevalence, natural history, functional effects and treatment. Clin Exp Optom 2005;88(6):365-375.

4. Hubel DH, Wiesel TN. The period of susceptibility to the physiological effects of unilateral eye closure in kittens. J Physiol 1970;206(2):419-436.

5. Berardi N, Pizzorusso T, Maffei L. Critical periods during sensory development. Curr Opin Neurobiol 2000;10(1):138-145.

6. Fronius $\mathrm{M}$, Cirina L, Ackermann $\mathrm{H}$, et al. Efficiency of electronically monitored amblyopia treatment between 5 and 16 years of age: new insight into declining susceptibility of the visual system. Vis. Res 2014;103:11-19.

7. Astle AT, Webb BS, McGraw P V. Can perceptual learning be used to treat amblyopia beyond the critical period of visual development? Ophthalmic Physiol Opt 2011;31(6):564573.

8. Levi DM, Li RW. Improving the performance of the amblyopic visual system. Philos Trans R Soc L. B Biol Sci 2009;364(1515):399-407.

9. Levi DM, Polat U. Neural plasticity in adults with amblyopia. Proc Natl Acad Sci U S A 1996;93(13):6830-6834.

10. Li RW, Ngo C, Nguyen J, Levi DM. Video-game play induces plasticity in the visual system of adults with amblyopia. PLoS Biol 2011;9(8):e1001135. 
11. Vedamurthy I, Nahum M, Huang SJ, et al. A dichoptic custom-made action video game as a treatment for adult amblyopia. Vis. Res 2015;114:173-187.

12. Xu JP, He ZJ, Ooi TL. Effectively reducing sensory eye dominance with a push-pull perceptual learning protocol. Curr Biol 2010;20(20):1864-1868.

13. Spiegel DP, Byblow WD, Hess RF, Thompson B. Anodal transcranial direct current stimulation transiently improves contrast sensitivity and normalizes visual cortex activation in individuals with amblyopia. Neurorehabil Neural Repair 2013;27(8):760-769.

14. Thompson B, Mansouri B, Koski L, Hess RF. Brain plasticity in the adult: modulation of function in amblyopia with rTMS. Curr Biol 2008;18(14):1067-1071.

15. Boroojerdi B, Battaglia F, Muellbacher W, Cohen LG. Mechanisms underlying rapid experience-dependent plasticity in the human visual cortex. Proc Natl Acad Sci U S A 2001;98(25):14698-14701.

16. Boroojerdi B, Bushara KO, Corwell B, et al. Enhanced excitability of the human visual cortex induced by short-term light deprivation. Cereb Cortex 2000;10(5):529-534.

17. Pitskel NB, Merabet LB, Ramos-Estebanez C, et al. Time-dependent changes in cortical excitability after prolonged visual deprivation. Neuroreport 2007;18(16):1703-1707.

18. Merabet LB, Hamilton R, Schlaug G, et al. Rapid and reversible recruitment of early visual cortex for touch. PLoS One 2008;3(8):e3046.

19. Lunghi C, Burr DC, Morrone C. Brief periods of monocular deprivation disrupt ocular balance in human adult visual cortex. Curr. Biol. 2011;21(14):R538-R539.

20. Lunghi C, Burr DC, Morrone MC. Long-term effects of monocular deprivation revealed with binocular rivalry gratings modulated in luminance and in color. J Vis 2013;13(6) 
22. Zhou J, Clavagnier S, Hess RF. Short-term monocular deprivation strengthens the patched eye's contribution to binocular combination. J Vis 2013;13(5)

23. Zhou J, Reynaud A, Hess RF. Real-time modulation of perceptual eye dominance in humans. Proc Biol Sci 2014;281(1795)

24. Turrigiano G. Homeostatic synaptic plasticity: local and global mechanisms for stabilizing neuronal function. Cold Spring Harb Perspect Biol 2012;4(1):a005736.

25. Lunghi C, Emir UE, Morrone MC, Bridge H. Short-Term monocular deprivation alters GABA in the adult human visual cortex. Curr. Biol. 2015;25(11):1496-1501.

26. Lunghi C, Sale A. A cycling lane for brain rewiring. Curr Biol 2015;25(23):R1122-3.

27. Baroncelli L, Bonaccorsi J, Milanese $\mathrm{M}$, et al. Enriched experience and recovery from amblyopia in adult rats: impact of motor, social and sensory components. Neuropharmacology 2012;62(7):2388-2397.

28. Fu Y, Kaneko M, Tang $\mathrm{Y}$, et al. A cortical disinhibitory circuit for enhancing adult plasticity. Elife 2015;4:e05558.

29. Kaneko M, Stryker MP. Sensory experience during locomotion promotes recovery of function in adult visual cortex. Elife 2014;3:e02798.

30. Stryker MP. A Neural Circuit That Controls Cortical State, Plasticity, and the Gain of Sensory Responses in Mouse. Cold Spring Harb Symp Quant Biol 2014;79:1-9.

31. Heimel JA, van Versendaal D, Levelt CN. The role of GABAergic inhibition in ocular dominance plasticity. Neural Plast 2012;2011:391763.

32. Bangerter A. [From the practice for the practice]. Ophthalmologica 1953;125(4-5):398-405.

33. Andree $\mathrm{G}$. [The influence of inverse occlusion on fixation and function of amblyopic eyes]. Albr. Von Graefes Arch Klin Exp Ophthalmol 1966;170(3):257-264.

34. Malik SR, Gupta AK, Choudhry S, Sen DK. Red filter treatment in eccentric fixation. Br J 
Ophthalmol 1968;52(11):839-842.

395

396

397

35. Malik SR, Gupta AK, Grover VK. Occlusion therapy in amblyopia with eccentric fixation. Br J Ophthalmol 1970;54(1):41-45.

36. Noordeng V. Occlusion Therapy in Amblyopia with Eccentric Fixation. Arch Ophthalmol $1965 ; 73: 776-781$.

37. Lunghi C, Emir UE, Morrone MC, Bridge H. Short-Term Monocular Deprivation Alters GABA in the Adult Human Visual Cortex. Curr Biol 2015;25(11):1496-1501.

38. Zhou J, Baker DH, Simard M, et al. Short-term monocular patching boosts the patched eye's response in visual cortex. Restor Neurol Neurosci 2015;33(3):381-387.

39. Lunghi C, Morrone MC, Secci J, Caputo R. Binocular Rivalry Measured 2 Hours After Occlusion Therapy Predicts the Recovery Rate of the Amblyopic Eye in Anisometropic Children. Invest Ophthalmol Vis Sci 2016;57(4):1537-1546.

40. Zhou J, Thompson B, Hess RF. A new form of rapid binocular plasticity in adult with amblyopia. Sci Rep 2013;3:2638.

41. Niell CM, Stryker MP. Modulation of visual responses by behavioral state in mouse visual cortex. Neuron 2010;65(4):472-479.

42. Bullock T, Cecotti H, Giesbrecht B. Multiple stages of information processing are modulated during acute bouts of exercise. Neuroscience 2015;307:138-150.

43. Bullock T, Elliott JC, Serences JT, Giesbrecht B. Acute Exercise Modulates Feature-selective Responses in Human Cortex. J Cogn Neurosci 2016;1-14.

44. Baek SS. Role of exercise on the brain. J Exerc Rehabil 2016;12(5):380-385.

45. Ma CL, Ma XT, Wang JJ, et al. Physical exercise induces hippocampal neurogenesis and prevents cognitive decline. Behav Brain Res 2017;317:332-339.

46. Nie J, Yang X. Modulation of Synaptic Plasticity by Exercise Training as a Basis for Ischemic 
Stroke Rehabilitation. Cell Mol Neurobiol 2017;37(1):5-16.

47. de Assis GG, de Almondes KM. Exercise-dependent BDNF as a Modulatory Factor for the Executive Processing of Individuals in Course of Cognitive Decline. A Systematic Review. Front Psychol 2017;8:584.

48. Baroncelli L, Sale A, Viegi A, et al. Experience-dependent reactivation of ocular dominance plasticity in the adult visual cortex. Exp Neurol 2010;226(1):100-109.

49. Hanover JL, Huang ZJ, Tonegawa S, Stryker MP. Brain-derived neurotrophic factor overexpression induces precocious critical period in mouse visual cortex. J Neurosci 1999;19(22):RC40.

50. Huang ZJ, Kirkwood A, Pizzorusso T, et al. BDNF regulates the maturation of inhibition and the critical period of plasticity in mouse visual cortex. Cell 1999;98(6):739-755. 

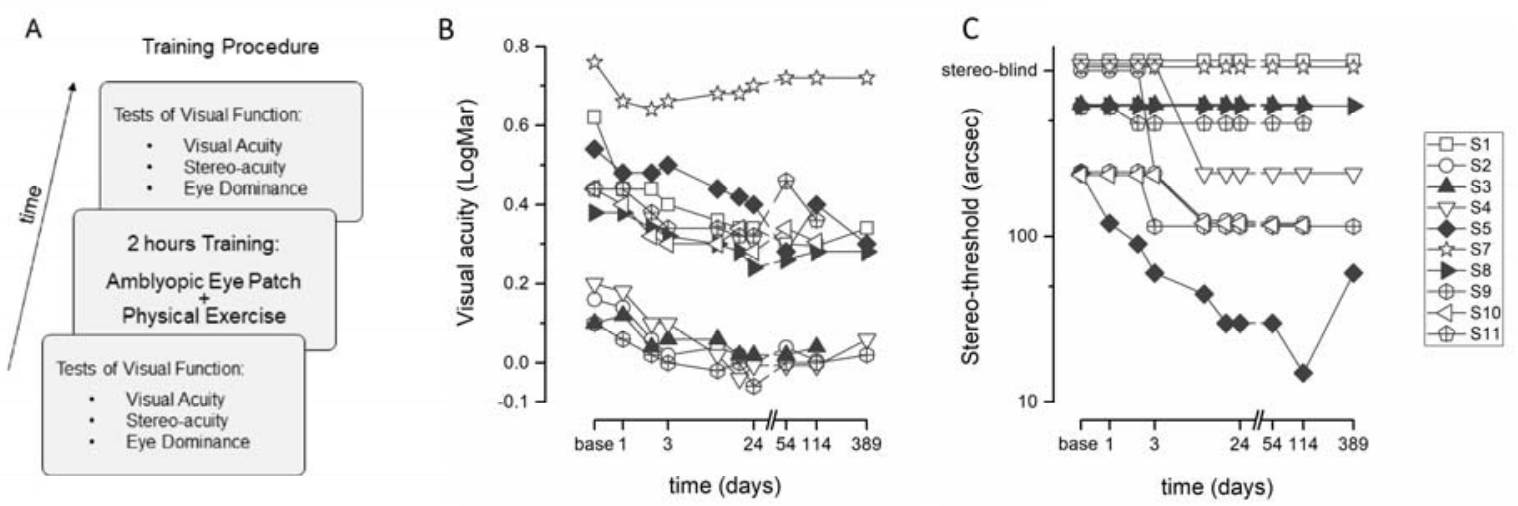

433

434

435

436

437

438

439

440

441
Figure 1. Training induces a recovery of visual acuity and stereo-threshold.

(A) 10 adult anisometropic amblyopes underwent a training paradigm in which short-term (2h) occlusion of the amblyopic eye was combined with physical exercise (intermittent cycling on a stationary bike, 10 minutes of activity and 10 minutes rest interleaved, heartrate between 110 and $120 \mathrm{bpm})$. During the $2 \mathrm{~h}$ training a movie was displayed in front of the stationary bike. Before and after each training session, sensory eye dominance (binocular rivalry between orthogonal gratings), visual acuity (ETDRS charts) and Stereo-thresholds (TNO test) were assessed for each patient. Visual acuity and stereo-thresholds were also assessed in follow-up measurements one, three and twelve months after the end of training. Each patient performed six $2 \mathrm{~h}$ training sessions: three on consecutive days during the first week of training, and one session per week during the following three weeks. (B) LogMar visual acuity plotted as a function of time from the beginning of training. To achieve a robust quantification of visual acuity, each point represents the average of three different ETDRS charts. Different symbols represent the different performances after each training session. Data points after the break in the abscissa represent visual acuity obtained in follow-up measurements performed one 54 days, 124 days and 395 days after the first training session. (C) Stereo-thresholds plotted as a function of time from the beginning of training. Stereothresholds were obtained using the TNO test. For subjects showing course stereopsis with the TNO test (S3, S8, S11), stereo-thresholds were obtained using the LANG stereo-test. 

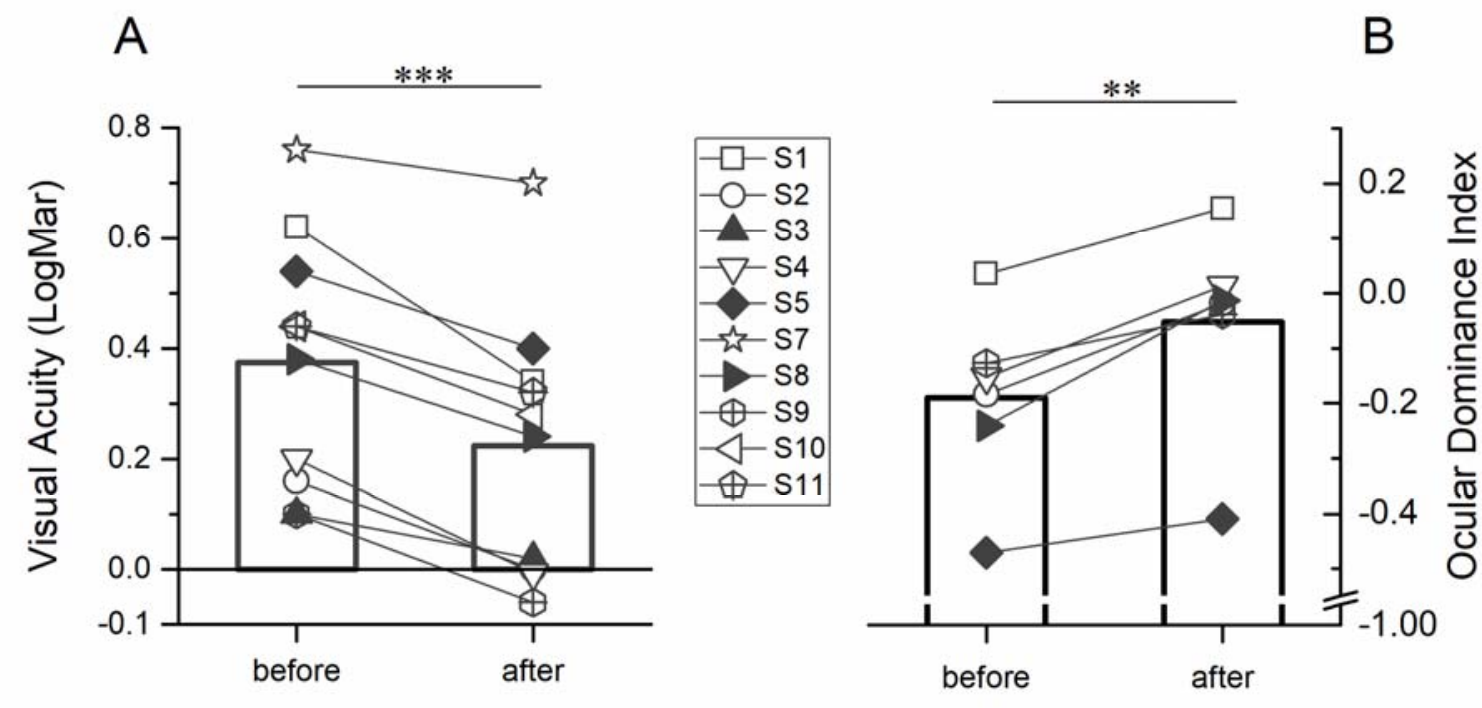

Figure 2. Visual acuity improvement and sensory eye dominance change after training.

(A) LogMar visual acuity measured before and after the four-week training is reported for each subject (different symbols for single subjects, bars: average visual acuity). Visual acuity exercise), averaged for each subject across training sessions (different symbols for single subjects, bars: average eye dominance). Sensory eye dominance is estimated as the contrast between the mean phase duration of the amblyopic (deprived) and non-amblyopic (non-deprived) eye in a binocular rivalry paradigm (Eq.1). The contrast of the rivalrous stimuli was adjusted before the training for each subject in order to observe perceptual alternations and eye dominance as balanced (close to the value 0) as possible (maximum contrast difference between the eyes: 100 $20 \%$, amblyopic - non amblyopic eye). Even after contrast adjustment, 4/10 patients did not perceive alternations in visual dominance during binocular rivalry (complete dominance of the non-amblyopic eye). Dominance of the amblyopic eye increased after short-term monocular deprivation combined with physical exercise (paired-samples t-test, $* *=p<0.001$ ). 

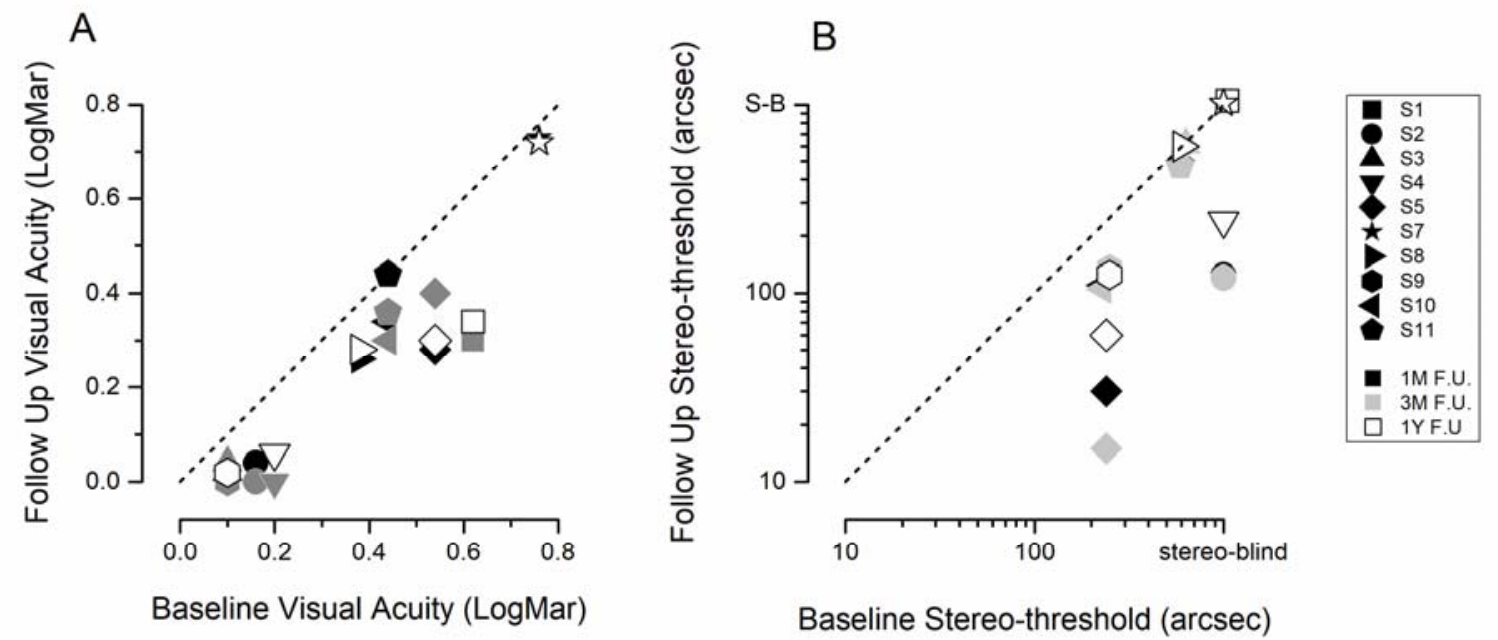

Figure 3. Visual acuity and stereo-threshold in follow-up measurements.

471 (A) Scatter plot of LogMar visual acuity measured before training ( $x$ axis) and in follow-up 472 measurements obtained one month (black symbols), three months (grey symbols) and one year 473 (white symbols) after the end of training (different symbols shapes represent different patients).

474 All points lie under the equality line indicating that the visual acuity improvement was maintained 475 in follow-up measurements. (B) Same as (A) but for stereo-thresholds: patients who improved 476 stereo-vision during the training maintained the improvement in follow-up measurements. 

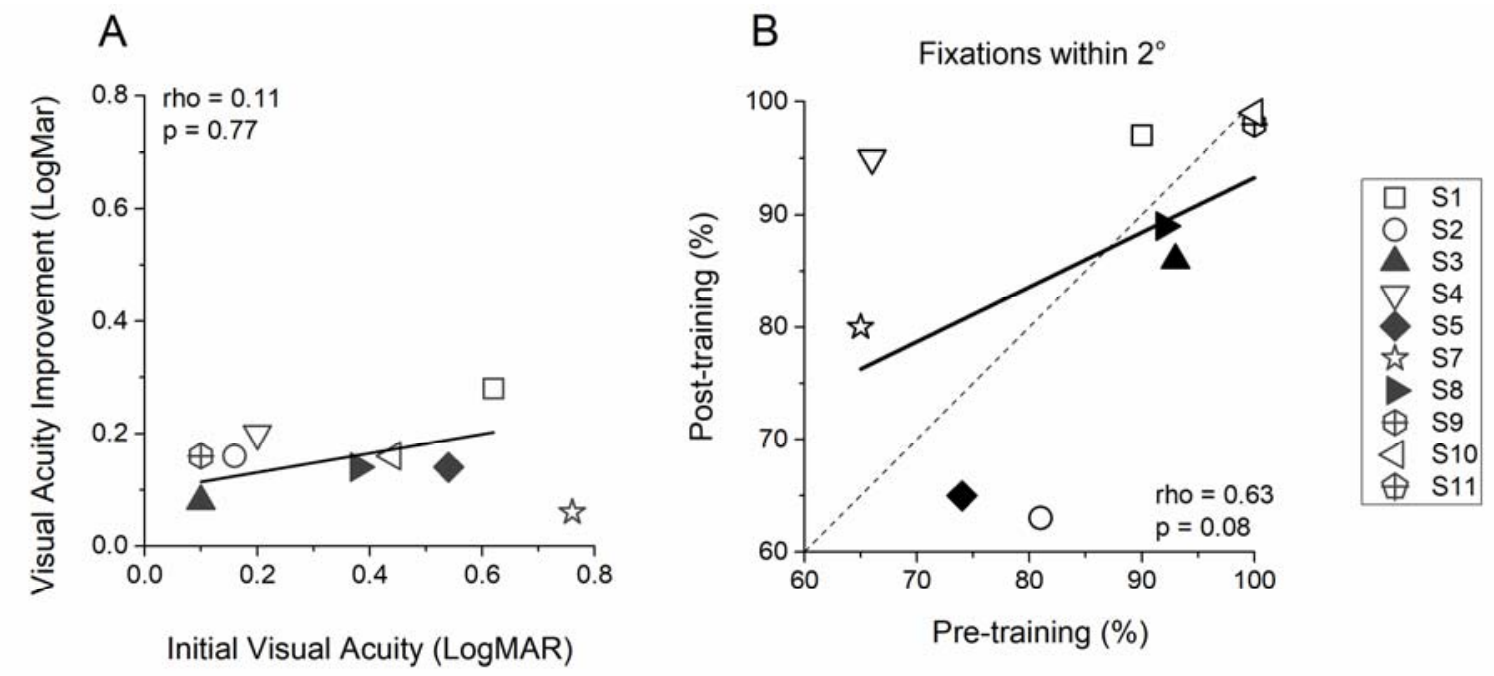

Figure 4. Correlations between anisometropia, visual acuity improvement and fixation quality.

(A) Scatter plot of the LogMar visual acuity measured for each subject (different symbols) before

training ( $x$ axis) and the difference between visual acuity measured after and before the 4-week

483 Scatter plot reporting the percentage of fixations falling within a 2-degree radius from the fixation in fixation quality is observed across subjects. 
A

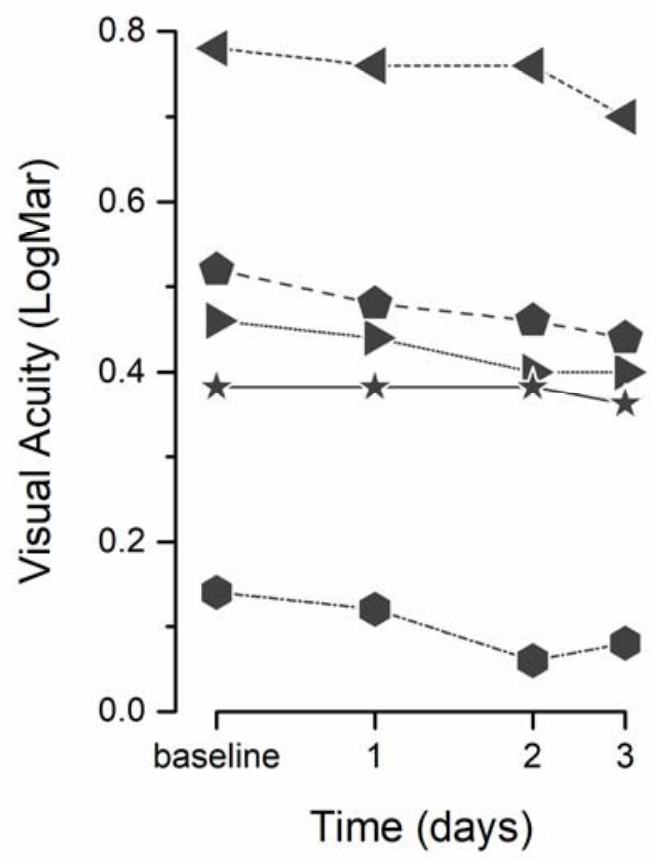

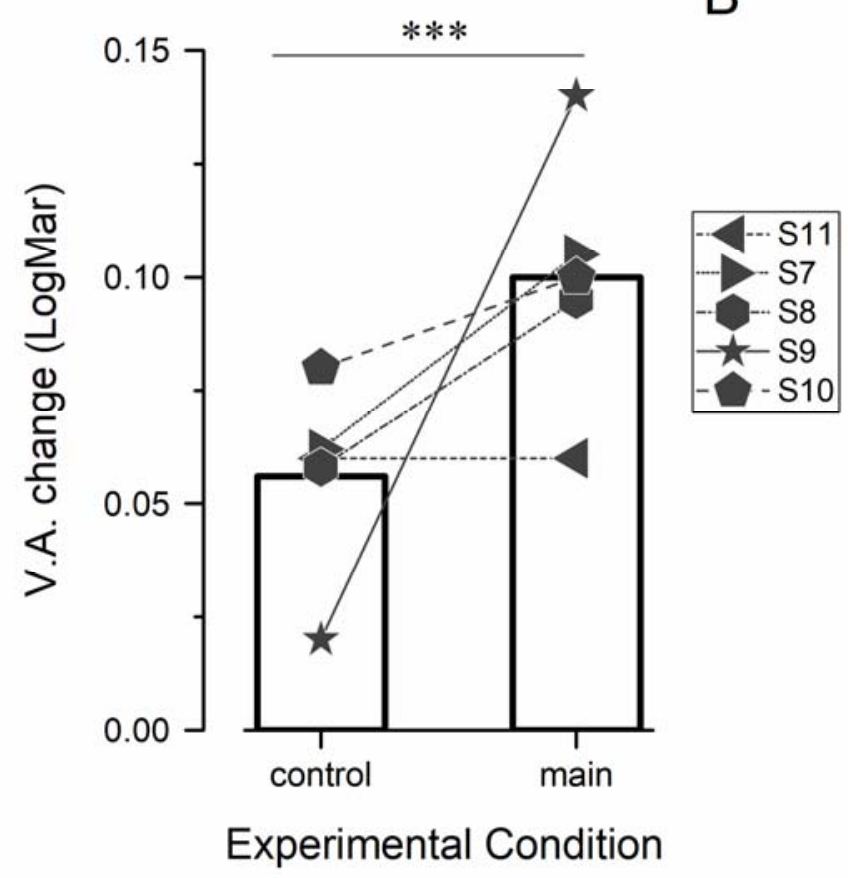

486

487

488

489

490

491

492

493

494

495

496

497

498

499

Figure 5. Control experiment results.

Five out of the 10 patients also performed a control experiment (at least one month before taking part in the main experiment). In three consecutive days, the non-amblyopic eye was patched without the simultaneous physical exercise performance. (A) LogMar visual acuity plotted as a function of time from the beginning of the control experiment (different symbols represent individual patient performance). Only a small improvement in visual acuity was observed in the control experiment. (B) LogMar stereo-threshold plotted as a function of time from the beginning of the control experiment. Stereo-thresholds did not change in the control experiment. (C) Comparison between the visual acuity improvement observed in the control experiment and after the first three days of training in the main experiment combining monocular occlusion and physical exercise (different symbols for single subjects, bars: average visual acuity). The visual acuity improvement is significantly larger (bootstrap sign-test, $* * *=p<0.001$ ) for the main experiment. 
Tables

\begin{tabular}{|c|c|c|c|c|c|c|c|c|}
\hline \multirow[t]{2}{*}{$\begin{array}{c}\text { Patient } \\
\text { ID }\end{array}$} & \multirow[t]{2}{*}{ Age/sex } & \multicolumn{2}{|c|}{ Refractive Error } & \multicolumn{2}{|c|}{ Visual Acuity (LogMar) } & \multirow[t]{2}{*}{$\begin{array}{l}\text { Stereoacuity } \\
\text { (ArcSec) }\end{array}$} & \multirow[t]{2}{*}{ Fixation AE } & \multirow[t]{2}{*}{ Treatment history } \\
\hline & & Right Eye & Left Eye & Right Eye & Left Eye & & & \\
\hline S1 & $28 / F$ & $+0.25+0.25 \times 75$ & $+2.25+2.25 \times 55$ & -0.06 & 0.62 & $\mathrm{~F}$ & $\begin{array}{l}\text { Central } \\
\text { unsteady }\end{array}$ & Patched at age 5 \\
\hline S2 & $29 / M$ & +0.00 & $-1.00 \times 60$ & -0.2 & 0.16 & $F$ & Central & No Treatment \\
\hline 53 & $27 / F$ & $-6.50-3.00 \times 10$ & $-0.25-0.75 \times 165$ & 0.1 & -0.14 & 600 & Central & Patched at age 11 \\
\hline S4 & $36 / F$ & $+0.25-7.00 \times 10$ & -1.00 & 0.2 & -0.2 & $F$ & Central & No Treatment \\
\hline S5 & $35 / M$ & +0.00 & $+3.00 \times 90$ & -0.2 & 0.54 & 240 & $\begin{array}{l}\text { Central } \\
\text { unsteady }\end{array}$ & No Treatment \\
\hline 57 & $34 / F$ & $-0.25 \times 180$ & $+2.00-1.50 \times 180$ & -0.1 & 0.78 & $F$ & Central & Patched at ages 7-10 \\
\hline S8 & $38 / F$ & $-3.25-1.50 \times 10$ & $-1.00-2.75 \times 180$ & 0.0 & 0.46 & 600 & Central & Patched at age 5 \\
\hline 59 & $40 / \mathrm{M}$ & +0.00 & +2.00 & -0.2 & 0.14 & 240 & Central & Patched at age 5 \\
\hline S10 & $34 / \mathrm{M}$ & +0.50 & $-0.50-0.50 \times 90$ & 0.38 & -0.2 & 240 & Central & Patched at ages 7-13 \\
\hline S11 & $28 / F$ & $+5.00+1.00 \times 80$ & +0.00 & 0.52 & -0.1 & 600 & $\begin{array}{c}\text { Central } \\
\text { unsteady }\end{array}$ & No Treatment \\
\hline
\end{tabular}

502 